\title{
Minocycline Protects Against the Degeneration of Retinal Neurons in Mice
}

\author{
Rui Guo* \\ Guangdong-Hongkong-Macau Institute of CNS Regeneration, Ministry of Education CNS Regeneration Collaborative \\ Joint Laboratory, Jinan University, Guangzhou, Guangdong Province, 510632, China
}

\begin{abstract}
Background and objectives: Microglia activation can cause degeneration of retinal ganglion cells (RGCs). This study aimed to investigate the potential therapeutic effect of minocycline on microglia activation-related degeneration of RGCs in both retinas after unilateral optic nerve crush (ONC) in the left eye of male adult C57BL/6 mice.

Methods: First, the primary degeneration of RGCs after unilateral ONC in the left eye and the secondary degeneration of RGCs in the contralateral eye were investigated. Second, microglia activation in both eyes was examined longitudinally at 1,5 and 14 days post-ONC. Finally, the effects of minocycline treatment on the primary or/and secondary RGC degeneration as well as the function of both retinas (estimated by flash electroretinogram) at 5 days post-ONC were analyzed.
\end{abstract}

Results: The results indicated that ONC induced the primary RGC degeneration, which was more severe than the secondary RGC degeneration and microglia activation in both eyes. Treatment with minocycline partially inhibited microglia activation, preserved the function of retinas in both eyes, and delayed the secondary degeneration of RGCs in the contralateral retina.

Conclusions: ONC caused RGC degeneration in both eyes of mice. Minocycline treatment delayed the secondary degeneration of RGCs and improved the function of both retinas post-ONC in mice, which were associated with inhibition of microglia activation.

Introduction

Glaucoma, characterized by the degeneration of retinal ganglion cells (RGCs), is the second leading cause of blindness in the world. ${ }^{1}$ Glaucoma can result in both primary and secondary degeneration. ${ }^{2}$ The early death of neurons and glial cells caused by

Keywords: Minocycline; Optic nerve crush; Retinal ganglion cells; Microglia; neuroprotection; Electroretinogram.

Abbreviations: Brn3a, brain-specific homeobox/POU domain protein 3A; ERG, electroretinogram; GCL, ganglion cell laye; Iba-1, ionized calcium binding adaptor molecule-1; INL, inner nuclear layer; IOH, intraocular hypertension; IPL, inner plexiform layer; ONC, Optic Nerve Crush; ONL, outer nuclear layer; ONT, optic nerve transection; OPL, outer plexiform layer; PBS, phosphate buffer saline; RGCs, retinal ganglion cells.

Received: May 20, 2020; Revised: June 04, 2020; Accepted: June 08, 2020

*Correspondence to: Rui Guo, Guangdong-Hongkong-Macau Institute of CNS Regeneration, Ministry of Education CNS Regeneration Collaborative Joint Laboratory, Jinan University, Guangzhou, Guangdong Province, 510632, China. Tel: $+86-020-$ 8522-3480, Fax: +86-020-8522-1343, E-mail: agogogray@gmail.com

How to cite this article: Guo R. Minocycline Protects Against the Degeneration of Retinal Neurons in Mice. J Explor Res Pharmacol 2020;5(4):61-72. doi: 10.14218/ JERP.2020.00015. the primary pathological events is termed primary degeneration. In addition, the death of the neuron and glial cells from the following toxic effects of primary degeneration is attributed to secondary degeneration. ${ }^{3}$ Studies have shown that damage to one eye can lead to secondary degeneration in the other eye, for example, unilateral high intraocular pressure in rats induces nerve inflammation, nerve fiber loss and apoptosis of RGCs in the bilateral eyes. ${ }^{4-6}$ Apart from the RGC degeneration, the degeneration of bipolar and/or photoreceptors was also demonstrated in the intraocular hypertension (IOH) model; specifically, the amplitudes of both a- and b- waves detected by flash electroretinogram (ERG) after injury reduced significantly, while the number of synaptic bands of photoreceptors and the thickness of outer nucleus and plexus layers of the same eye significantly decreased simultaneously. ${ }^{7}$ Hence, both primary and secondary degeneration can happen after damage to an eye, including both the degeneration of RGCs and the impairment of other neurons, such as bipolar cells and/or photoreceptors. Therefore, this study was designed to investigate both the primary and secondary degeneration of RGCs in mice.

Tetracyclines are a broad-spectrum class of antibiotics. Minocycline is a member of the semisynthetic tetracyclines and the most potent agent in the family. ${ }^{8}$ Minocycline can inhibit microglia 
activation, cell apoptosis and reactive oxygen species production. ${ }^{9}$ Previous studies have shown that minocycline can inhibit the progression of several neurological diseases in rodent models, such as depression, Parkinson's disease, Alzheimer's disease, and cerebral ischemia. ${ }^{10-13}$ Growing data also indicate that minocycline treatment can protect from the injury and death of neuronal cells in some retinal diseases. ${ }^{14-16}$ In animal studies of glaucoma, such as via the optic nerve transection (ONT) model and IOH model, minocycline treatment effectively delayed the death of RGCs in the injured eye,${ }^{17}$ indicating that minocycline inhibited the primary degeneration of RGCs. However, a study of the partial ONT model of rats claimed that minocycline delayed the secondary degeneration of RGCs in the inferior-nasal retinas rather than primary degeneration in the superior-nasal retinas. ${ }^{18}$ Thus, the role of minocycline treatment in retinopathy remains inconclusive and needs further investigation.

In many studies related with eye diseases, microglia activation were believed to induce the death of RGCs and a significant negative correlation was found between the number of surviving RGCs and the numbers of activated microglia in the mice administrated using the acute $\mathrm{IOH}, \mathrm{ONT}$ and optic nerve crush (ONC) model. ${ }^{19,20}$ The ONC model has been widely used to investigate the pathogenesis of $\mathrm{RGC}$ death and to explore new protective treatments for glaucoma. ${ }^{21,22}$ Although minocycline treatment has shown to partially protect from neuronal death in the crushed eye in $\mathrm{ONC}$ mice, ${ }^{23}$ it is unclear whether minocycline treatment can attenuate microglia activation in the contralateral eye post$\mathrm{ONC},{ }^{24}$ which can cause the secondary degeneration of RGCs. Thus, this study was designed to explore the degeneration of primary and secondary RGCs and to investigate microglia activation, RGC survival, and retina function (flash ERG examination) in both eyes of mice.

\section{Materials and methods}

\section{Animals}

Male C57/BL6 mice (5 weeks-old, 18-22 g) were purchased from Guangdong Province Medical Laboratory Animal Center in Guangzhou, China. All animals were housed in a temperaturecontrolled room with a 12-hour light/12-hour dark cycle and were supplied with food and water. A total of 32 mice were used in these experiments, with 17 mice used for determination of the surviving RGCs and microglia activation in the normal group (4 mice), ONC 1-day group (3 mice), ONC 5-day group (4 mice) and ONC 2-week group (6 mice), and 15 mice used for investigation on the effects of minocycline in the normal group (4 mice), phosphate buffer saline (PBS) (0.01 M, pH 7.4) group (4 mice) and minocycline group (7 mice). All animal procedures were performed according to the ARRIVE guidelines and were approved by the Animal Protection and Use Committee of Jinan University (Protocol Number: 2018136). All efforts were taken to minimize the number of animals used and their suffering.

\section{Mouse ONC model}

The ONC mouse model has been widely used for studying the pathogenesis and therapeutic strategies for glaucoma. ${ }^{21}$ Briefly, the mice were anesthetized with tribromoethanol $(0.2 \mathrm{~mL} / 10 \mathrm{~g}$ body weight of $1.25 \%$ solution) and subjected to topical anesthetics (Oxybuprocaine Hydrochloride Eye Drops; Santen Pharma- ceutical, Japan) to their left eyeballs. The temporal conjunctiva of the mice was incised, and the optic nerve was exposed without damaging the blood sinusoid and clamped with tweezers (02095-PO; Dument, Switzerland) at $0.5 \mathrm{~mm}$ behind the optic disc for $10 \mathrm{~s}$. Subsequently, the incision was covered and the surface of the cornea was treated with erythromycin eye ointment to prevent postoperative infection.

\section{Minocycline injection}

The ONC mice were randomized and injected intraperitoneally with vehicle PBS or minocycline $(45 \mathrm{mg} / \mathrm{kg}$; Sigma Aldrich, USA), using an effective dose described previously, ${ }^{25}$ given at 2 days before and daily or every $12-\mathrm{h}$ after the $\mathrm{ONC}$, until animal euthanasia, after which their retinas were collected for further analysis. The experimental protocol is illustrated in Figure 1a.

\section{Immunofluorescence}

The mice were killed with an overdose of anesthetics and perfused with cold $4 \%$ paraformaldehyde in PBS. The retinas were then dissected out and postfixed in the same fixative for $1 \mathrm{~h}$. Each intact retina was collected as a whole-mount and cut into four quadrants (the superior temporal, inferior temporal, superior nasal and inferior nasal parts) for subsequent analysis by immunofluorescence. ${ }^{16}$ In brief, RGCs and microglia were stained with goat anti-brain-specific homeobox/POU domain protein $3 \mathrm{~A}$ (Brn3a) antibody (1:1,000; Santa Cruz Biotechnology, USA) and rabbit anti-ionized calcium binding adaptor molecule-1 (Iba-1) antibody (1:800; Wako, Japan), respectively. Secondary antibodies were Alexa 488 or 594-conjugated donkey anti-goat and donkey anti-rabbit (1:1,000; Life Technologies, USA), followed by nuclear-staining with 4', 6-diamidino-2-phenylindole (DAPI) (1:2,000; Electron Microcopy Sciences, USA) before the retinal flat-mount.

\section{Counting of RGCs}

Five photographs $\left(200 \times 200 \mu \mathrm{m}^{2}\right)$ were captured in each quadrant along the median line, starting from the outer edge of the optic disc to the border of each part and taken at $500 \mu \mathrm{m}$ intervals at $400 \times$ magnification. The numbers of surviving RGCs were counted using ImageJ software (version 1.41; National Institutes of Health, USA). Then, the density values of RGCs $\left(\mathrm{mm}^{2}\right)$ averaged from the total 20 images of each retina were used for comparing the differences among/between various groups.

\section{Microglia cell analysis}

In the retina, anti-Iba-1-stained microglia were observed by means of a laser confocal microscope and located within the following three layers: ganglion cell layer (GCL); inner plexiform layer (IPL); and outer plexiform layer (OPL) (green color, Fig. 1b). The location of GCL was first determined by the nuclei and cell bodies of RGCs stained by DAPI (blue color) and Brn3a (red color), which appeared arranged in just a single layer on the top (Fig. 1b). The location of the inner nuclear layer (INL) and outer nuclear layer $(\mathrm{ONL})$ was determined by the multilayered nuclei stained by DAPI; the INL was closer to the GCL and the ONL was farther from the GCL, with more nucleus layers than INL as well (Fig. 
a<smiles>[C]=CI</smiles><smiles>[C]=CI</smiles><smiles>C=[Te]</smiles><smiles>[C]=CI</smiles><smiles>[C]=CI</smiles><smiles>[C]=CI</smiles><smiles>[C]=CI</smiles><smiles>[C]=CI</smiles>

ONC

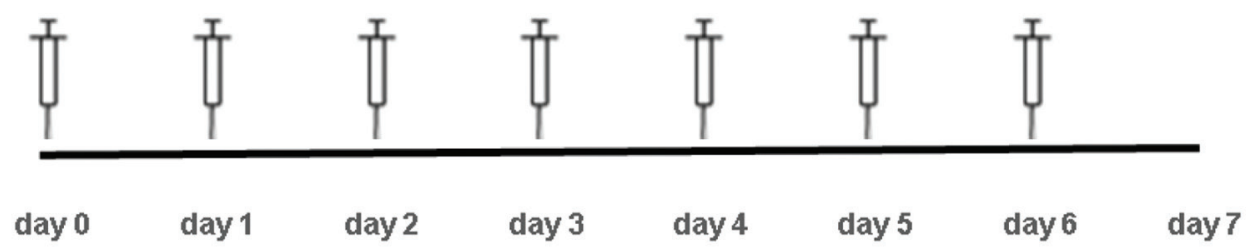

b
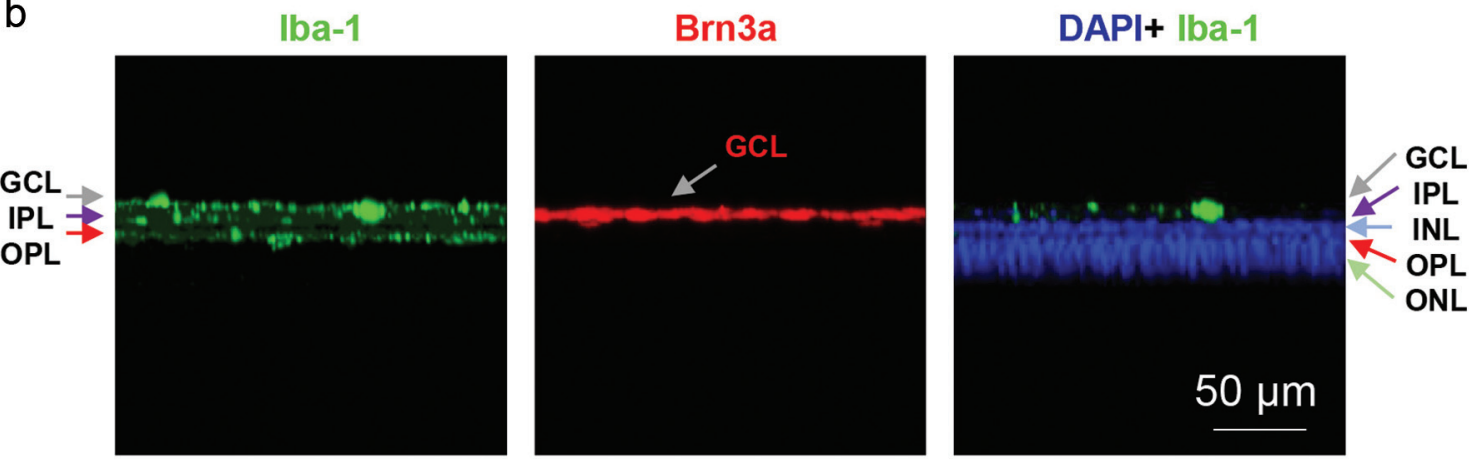

C

Iba-1
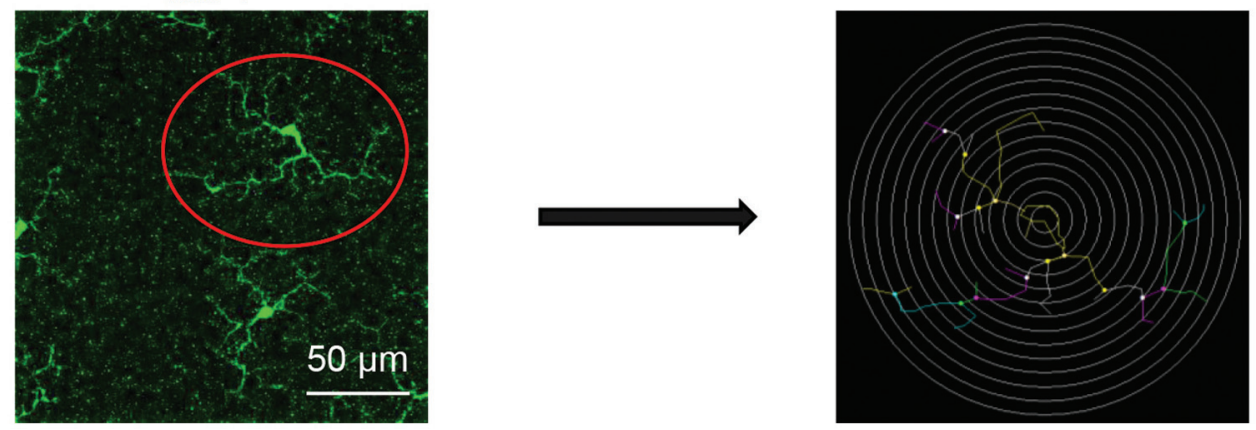

Fig. 1. Injection schedule of minocycline and methods of microglia analysis. (a) The injection was performed in a frequency of twice a day with an interval of 12 hours (one at 8 o'clock and the other at 20 o'clock), except for the last day when only one-time injection was given in the morning. The injection was started 2 days before ONC and continued every day until 5 days post-ONC. (b) Scanning of DAPI, Iba- 1 and Brn3a staining layers of the mouse retina at a 200x magnification. The three layers stained with Iba-1 (green color) from top to bottom are: GCL, IPL and OPL (as shown by the gray arrow, purple arrow and yellow arrow, respectively). The GCL stained with Brn3a (red color). The three layers stained with DAPI (blue color) from top to bottom are: GCL, INL and ONL, respectively (as shown by the gray arrow, blue arrow and green arrow, respectively). (c) Neurolucida 360 was used to reconstruct the morphology of retinal microglia cells, i.e. a microglia cell in the OPL 5 days post-ONC is shown on the left and the reconstructed image is shown on the right. Neurolucida Explore was used to analyze the reconstructed cells to obtain relevant information about cell body circumference, cell body area, node number, total number of branches, total branch length, and average branch length.

1b). The IPL was between the GCL and the INL, the OPL was between the INL and the ONL, and the microglia (green color) existed in the GCL, IPL and OPL layers (Fig. 1b).

Post-ONC, the changes in morphology of microglia in each layer were observed under a $20 \times$ objective and qualitatively analyzed. In addition, the quantitative analysis was conducted by ImageJ software. As it was difficult to count the specific number of microglia in the GCL due to the superposition of the microglial cell bodies and branches, the area of the Iba-1-positive cell bodies in the GCL in each photograph was manually recorded for each ani- mal and the values of all the photographs as the total cell area (Fig. $2 a, c$ and Fig. 3a, c). The numbers of microglia in the IPL and OPL were counted, given that the Iba-1-positive cells were separated by each other and could be discerned clearly in these two layers (Fig. 2a, c and Fig. 3a, c).

Apart from the analysis mentioned above which had been carried out for each animal, additional quantitative analysis was used to further investigate the morphological changes caused by minocycline at a $400 \times$ magnification. In the PBS and minocycline groups, the following parameters of microglia cells in the 
a $\mathrm{GCL}$
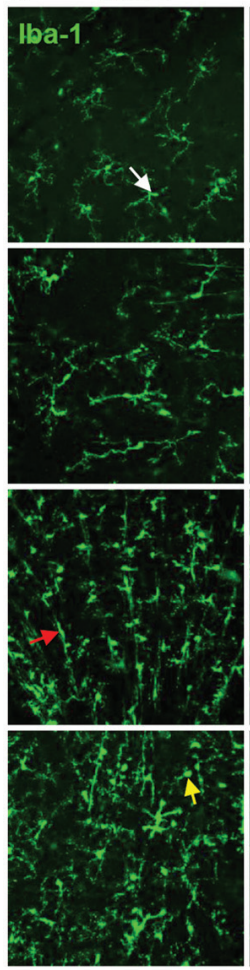

b

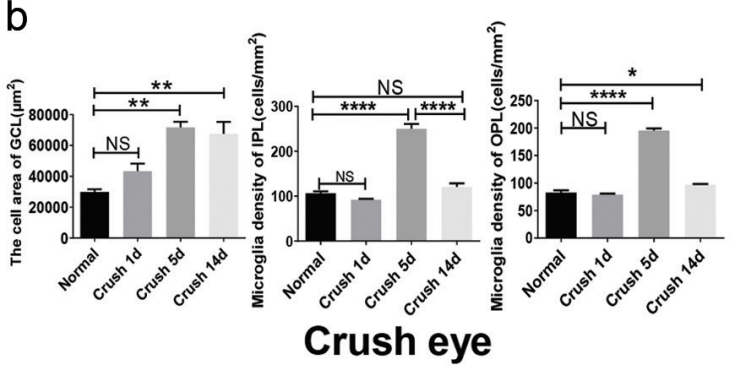

IPL

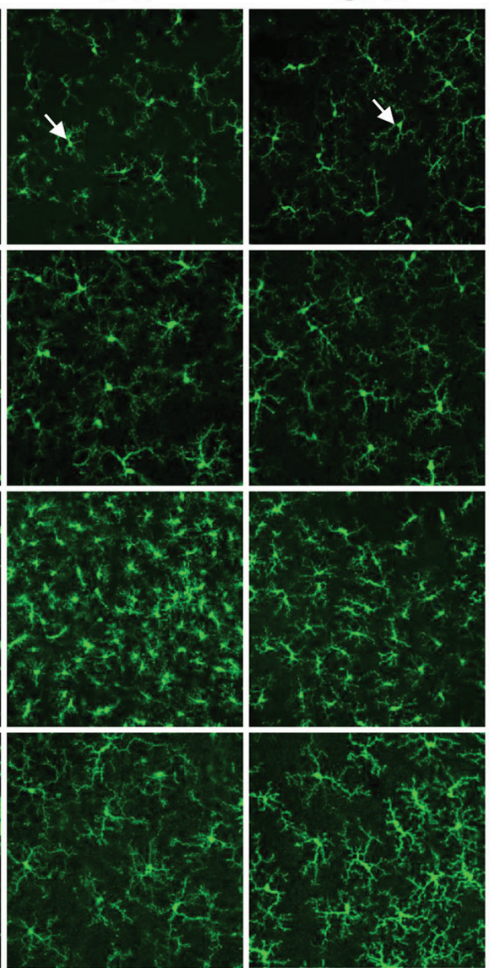

C $\quad \mathrm{GCL}$


d

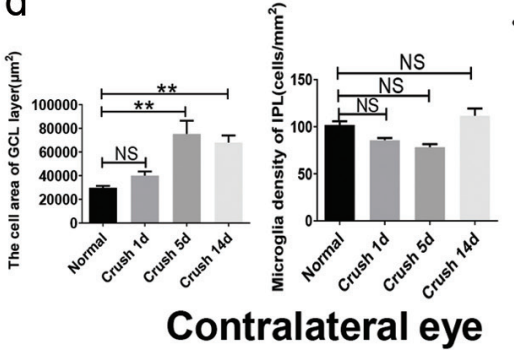

IPL

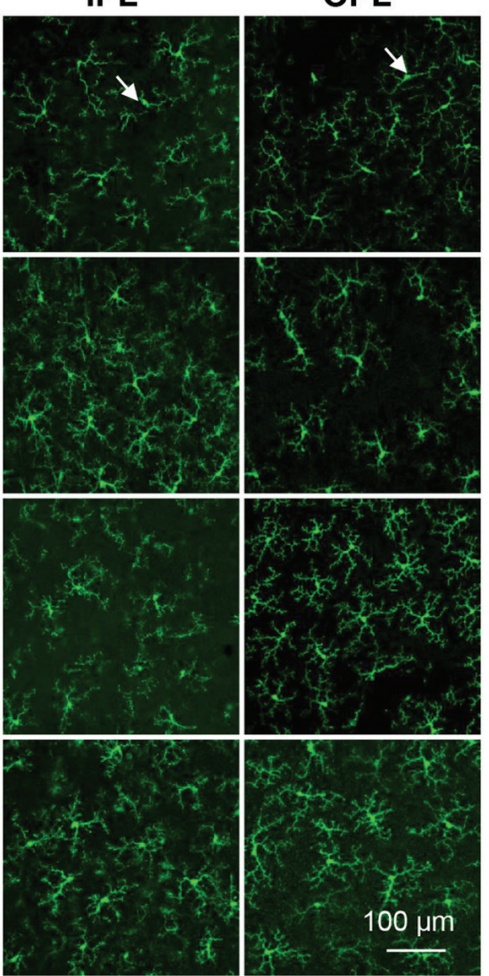

OPL

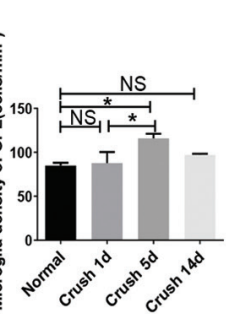

Fig. 2. Typical immunofluorescence staining images and the analysis of microglial cells shown by Iba1 staining in different retinal layers in both eyes of the normal mice and the mice at days 1, 5 and 14 post-ONC. (a) The typical immunofluorescence staining images illustrate the microglial cells in the retinal layer in the crushed eye of the normal mice and the mice at days 1, 5 and 14 post-ONC, as indicated. Post-ONC, microglia cells in the GCL, IPL and OPL gradually grew in size and branches became shorter and thicker over time. (b) The total area of microglia cell bodies in the GCL showed no significant difference at day 1 post-ONC but increased significantly at days 5 and 14 post-ONC. The number of microglia cells in the IPL showed no significant difference at 1 day and 14 days post-ONC, but increased significantly at 5 days post-ONC; the number of microglia in the OPL showed no significant difference at 1 day post-ONC, but increased significantly at 5 days and 14 days post-ONC. (c) The typical immunofluorescence staining images illustrate the microglial cells in the retinal layer in the contralateral eye of the normal mice and the mice at 1, 5, 14 days post-ONC, respectively. (d) The total area of microglia cell body in the GCL showed no significant difference at 1 day post-ONC but a significant increase at 5 days and 14 days post-ONC. The number of microglia in the IPL showed no significant difference at 1 day, 5 days and 14 days post-ONC. The number of microglia in the OPL showed no significant difference at 1 day and 14 days post-ONC but a significant increase at 5 days post-ONC. The data were tested by one-way ANOVA, followed by Tukey's multiple comparison test. The data are expressed as mean \pm standard error of the mean. NS, no significant difference. ${ }^{*} p<0.05,{ }^{* *} p<0.01, * * * * p<0.0001$. The number of animals in the normal, 1 day postONC, 5 day post-ONC and 2 week post-ONC groups were $4,3,4$ and 6 , respectively.

IPL and OPL were further quantified by Neurolucida software (version 2.7; Micro Brightfield, USA) after the photographs had been taken. The Neurolucida 360 tool was used to reconstruct the morphology of retinal microglia cells (Fig. 4a, c, e, g). Neurolucida Explore was used to analyze the reconstructed cells to obtain relevant information, including cell body circumference, cell body area, node number, total number of branches, total branch length, and average branch length of each cell in each photograph. The average values of each animal were used for comparison.

\section{ERG}

ERG was performed essentially as described previously. ${ }^{26}$ Briefly, the mice were dark adapted overnight; the animals were then anesthetized with tribromoethanol $(0.2 \mathrm{~mL} / 10 \mathrm{~g}$ body weight of $1.25 \%$ solution) and placed on a heated platform at $37{ }^{\circ} \mathrm{C}$. Pupils were 


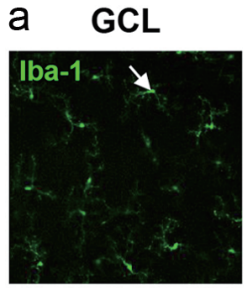

IPL
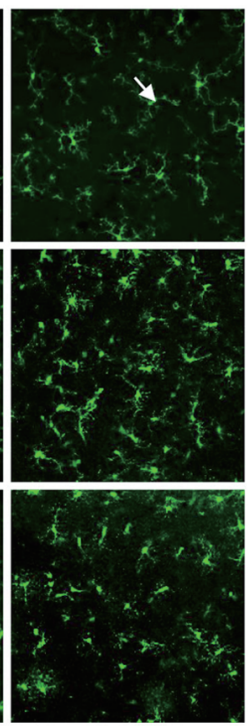

b

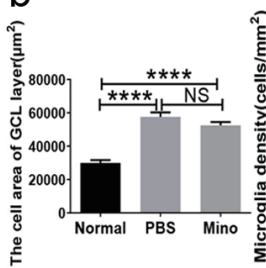

$\stackrel{8}{\stackrel{2}{5}}$
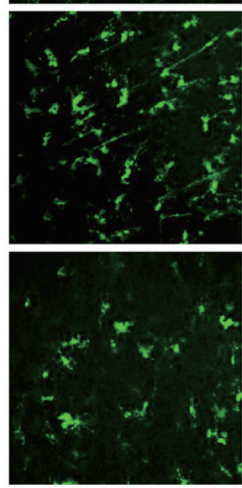

Crush eye
C
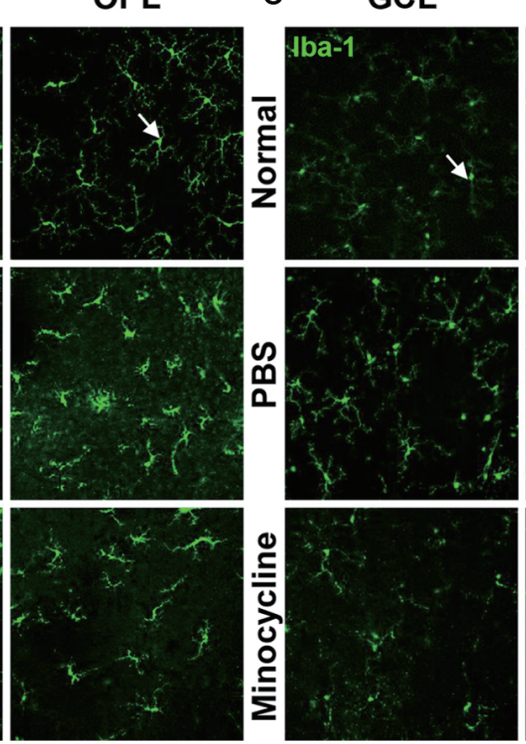

d

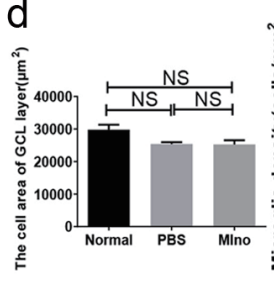

Contralateral eye

Fig. 3. Typical immunofluorescence staining images and the analysis of microglial cells shown by lba1 staining in different retinal layers in both eyes in the normal mice, PBS-treated ONC mice and minocycline-treated ONC mice. (a) The typical immunofluorescence staining images illustrate the microglial cells in the retinal layer in the crushed eye of the normal mice, PBS-treated ONC mice and minocycline-treated ONC mice. (b) In the crushed eye, compared with the eye of normal mice, the total area of microglia cell body in the GCL and the number of microglia in the IPL was increased significantly in the PBS-treated ONC mice and minocycline-treated ONC mice but there was no significant difference between the PBS-treated ONC mice and minocycline-treated ONC mice. There was a significant difference in the density of microglia in the OPL between the normal mice and PBS-treated ONC mice. There was no significant difference between the normal mice and minocycline-treated ONC mice. (c) The typical immunofluorescence staining images illustrate the microglial cells in the retinal layer in the contralateral eye of the normal mice, PBS-treated ONC mice and minocycline-treated ONC mice. (d) In the contralateral eye, compared with the normal mice, the total area of microglia cell body in the GCL and the number of microglia in the IPL showed no significant difference between the normal mice, PBS-treated ONC mice and minocycline-treated ONC mice. There was a significant difference in the density of microglia in the OPL between the normal mice and PBS-treated ONC mice. There was no significant difference between the normal mice and minocycline-treated ONC mice. The data were tested by one-way ANOVA followed by Tukey's multiple comparison test. The data are expressed as mean \pm standard error of the mean. NS, no significant difference. ${ }^{*} p<0.05,{ }^{*} p<0.01$, and $* * * * p<0.0001$. The number of animals in the normal group, PBS group and minocycline group were 4 , 4 and 7 , respectively.

dilated with Compound Tropicamide Eye Drops (Santen Pharmaceutical, China). ERGs were recorded with gold-plated wire loop electrodes contacting the corneal surface as the active electrode. Stainless steel needle electrodes were inserted in the skin near the eye and in the tail serving as reference and ground leads, respectively. Following adaptation, animals were stimulated with green flashes of graded intensities of $0.1,1.0$ and $10.0 \mathrm{~cd} . \mathrm{s} / \mathrm{m}^{2}$, followed by light adapted for $5 \mathrm{~m}$ under bright green background $(20 \mathrm{~cd} /$ $\mathrm{m}^{2}$ ) prior to recording of photopic responses to green flashes of $10.0 \mathrm{~cd} . \mathrm{s} / \mathrm{m}^{2}$.

\section{Statistical analysis}

All data are presented as mean \pm standard error of the mean. Statistical analysis was performed by GraphPad Prism (version 6.0;
GraphPad Software, USA). The data between two groups were compared using unpaired $t$-test and the data among multiple groups were compared using one-way ANOVA and post hoc Tukey's multiple comparison test. Statistical significance was defined as $p<0.05$.

\section{Results}

\section{Survival of RGCs post-ONC in mice}

In the normal C57 mice, the mean density of RGCs was 3,993 \pm $94 \mathrm{RGCs} / \mathrm{mm}^{2}$ in the left eye and $4,100 \pm 113 \mathrm{RGCs} / \mathrm{mm}^{2}$ in the right eye (Fig. 5a). At day 1, 5 and 14 post-ONC, the corresponding mean densities of surviving RGCs were 3,667 $\pm 55 \mathrm{RGCs} /$ 

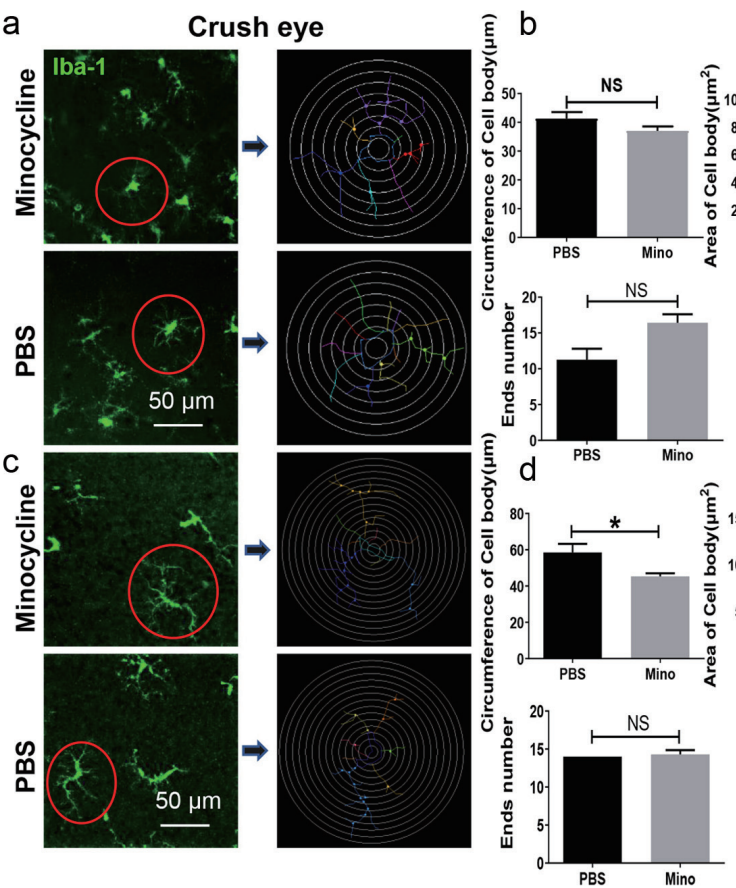

e
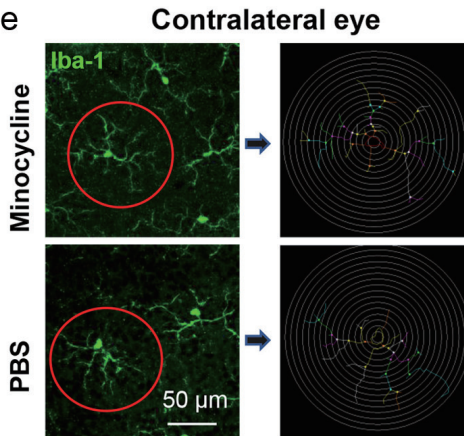

g


IPL
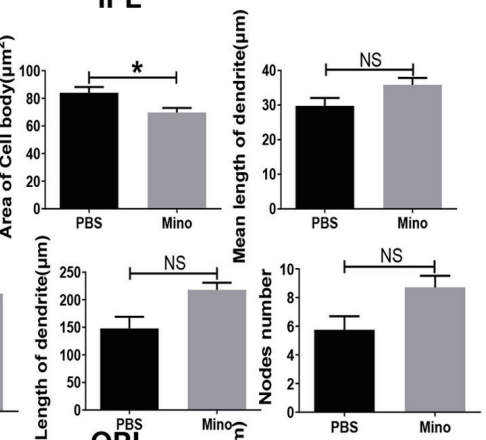

OPL
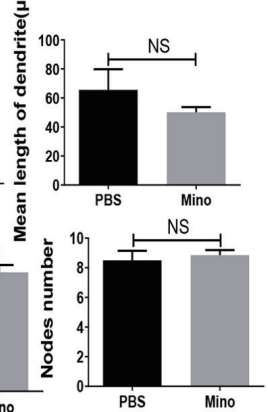

IPL


Fig. 4. Morphological changes of microglial cells shown by lba1 staining in the IPL and OPL in both eyes observed under a microscope with a 400x magnification. (a) Neurolucida software was used to reconstruct and compare the morphology of microglia in the IPL of the crushed eyes of mice; the original images are on the left, and the right side shows reconstructions. (b) Microglia cells in the IPL of the crushed eye of the PBS-treated ONC mice had larger cell area than that that of the minocycline-treated ONC mice. (c) Neurolucida software was used to reconstruct and compare the morphology of microglia in the OPL of the crushed eye of mice; the original images are on the left, and the right side shows reconstructions. (d) Microglia cells in the OPL of the crushed eye of the PBS-treated ONC mice had larger cell area than those in the minocycline-treated ONC mice. (e) Neurolucida software was used to reconstruct and compare the morphology of microglia in the IPL of the contralateral eye of mice; the original images are on the left, and the right side shows reconstructions. (f) Microglia cells in the IPL of the contralateral eye of the PBS-treated ONC mice had longer cell circumference, less node number and ends' number, and shorter length of dendrite and mean length of dendrite than those in the minocycline-treated ONC mice. (g) Neurolucida software was used to reconstruct and compare the morphology of microglia in the OPL of the contralateral eye of mice; the original images are on the left, and the right side shows reconstructions. (h) Microglia cells in the OPL of the contralateral eye of the PBS-treated ONC mice had longer cell circumference and larger cell area than those in the minocycline-treated ONC mice. The data were tested by unpaired t-test. The data were expressed as mean \pm standard error of the mean. NS, no significant difference. ${ }^{*} p<0.05$ and ${ }^{* *} p<0.01$. The number of animals in the normal group, PBS group and minocycline group were $4,4,7$, respectively. 
a

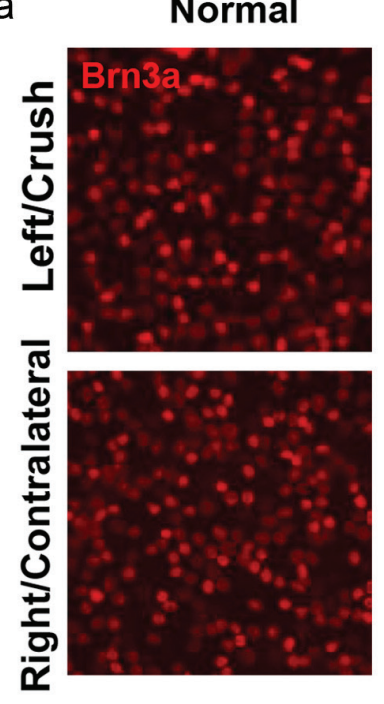

Crush 1d
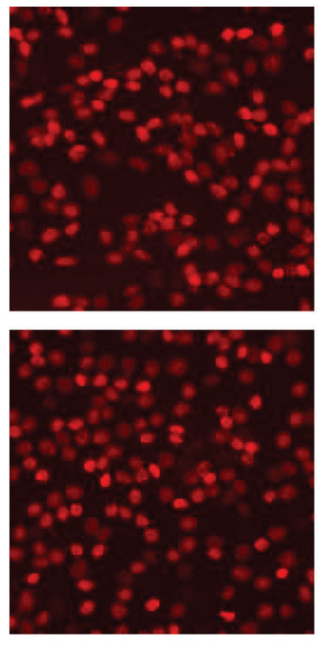

Crush 5d
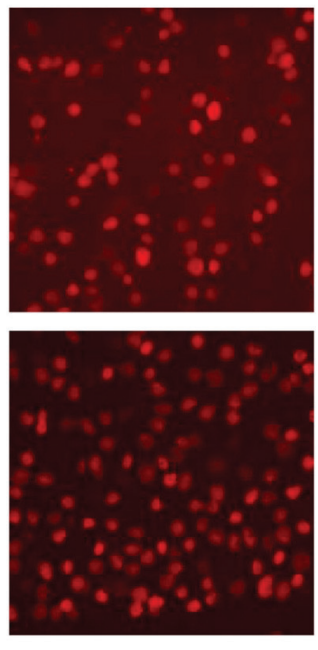

\section{Crush 14d}
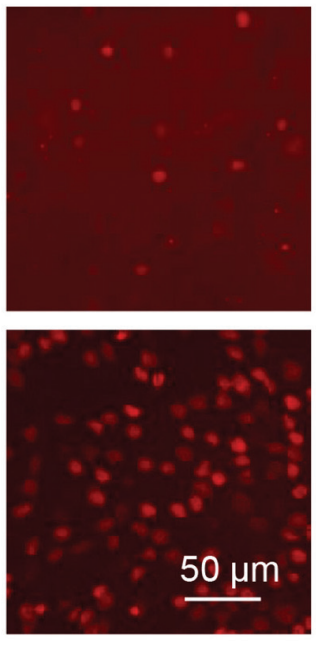

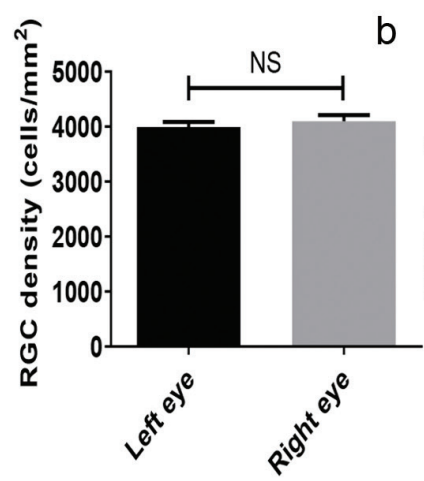

Normal eye

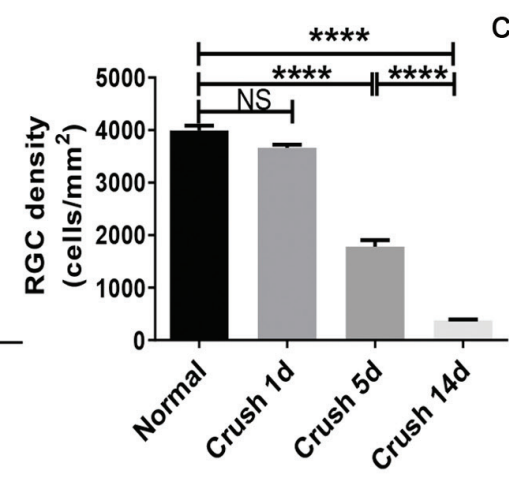

Crush eye



Contralateral eye

e

\section{Survival percent of RGCs}

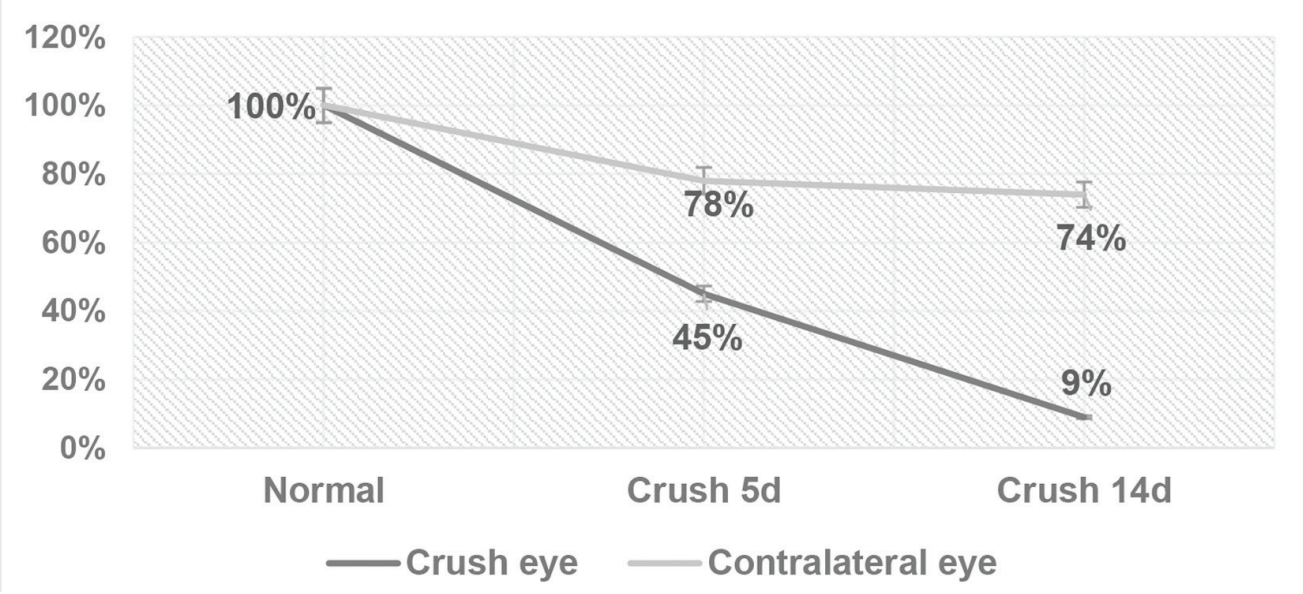

Fig. 5. Number of surviving RGCs was counted as Brn3a-positive cells in the healthy mice and ONC mice at different time-points post-ONC. (a) The typical immunofluorescent staining images of the RGCs (Brn3a-positive, red color) in the healthy and ONC mice. (b-d) Quantitative analysis of RGCs. (e) The percentages of surviving RGCs in the crushed and contralateral eyes at 5 and 14 days post-ONC. The mean number of RGCs in normal mice were set to $100 \%$. The data in (b) were assessed by unpaired $t$-test. The data in (c, d) were assessed by one-way ANOVA and post hoc Tukey's multiple comparison test. The data shown are representative images (magnification $\times 400$ ) and expressed as mean \pm standard error of the mean. NS, no significant difference. ${ }^{* *} p<0.01$, ${ }^{* * *} p<0.001$, and ${ }^{* * * *} p<0.0001$. The number of animals in the normal, 1-day ONC, 5-day ONC and 2-week ONC groups were $4,3,4$ and 6, respectively. 
$\mathrm{mm}^{2}, 1,781 \pm 124 \mathrm{RGCs} / \mathrm{mm}^{2}$ and $375 \pm 196 \mathrm{RGCs} / \mathrm{mm}^{2}$ in the left crushed eye of mice, while those in the right/contralateral eye were 3,900 $\pm 190 \mathrm{RGCs} / \mathrm{mm}^{2}, 3,195 \pm 123 \mathrm{RGCs} / \mathrm{mm}^{2}$ and 3,046 $\pm 145 \mathrm{RGCs} / \mathrm{mm}^{2}$, respectively (Fig. 5a). There was no significant difference in the numbers of RGCs between the left and right eye of normal mice (Fig. 5b). In the crushed eye, the number of RGCs decreased significantly at days 5 and 14 post-ONC but not day 1 post-ONC compared with the normal left eye (Fig. 5c), and the percentage of surviving RGCs were $45 \pm 3 \%$ and $9 \pm 1 \%$ at days 5 and 14 post-ONC, respectively (Fig. 5e). In the contralateral eye, the numbers of RGCs decreased significantly at days 5 and 14 post-ONC but not day 1 post-ONC compared with the normal right eye (Fig. 5d). There was no significant difference in the numbers of surviving RGCs in contralateral eye between days 5 and 14 post-ONC (Fig. 5d), and the percentage of surviving RGCs were $75 \pm 3 \%$ and $74 \pm 4 \%$ at days 5 and 14 post-ONC, respectively (Fig. 5e).

\section{Qualitative and quantitative analyses of retinal microglia post- ONC}

First, the morphological changes of microglial cells post-ONC were observed. During the transition from resting state under normal condition to active state after injury, the microglia cells were observed to undergo significant morphological changes in the cell body and the process. Microglia cells in the GCL, IPL and OPL of normal mice were characterized by small cell bodies and slender branches, as shown by the white arrows in Figure 2a and c. During the timeline of days 1,5 and 14 post-ONC, the cell bodies of microglia cells in the GCL, IPL and OPL of the injured eye gradually grew larger and the branches became shorter and thicker over time. In the IPL at day 5 post-ONC and the OPL at day 14 post-ONC, the microglia cells formed a dense mosaic pattern (Fig. 2a). The author found that two different shapes of microglia appeared post-ONC, i.e. the rod-shaped cell body with either branch at ends, as shown by the red arrow in Figure 2a, and the amoeba-like cells without protrusions, as shown by the yellow arrow in Figure 2a. During the timeline of days 1, 5 and 14 post-ONC, the cell bodies of microglia cells in the GCL, IPL and OPL of the contralateral eye also grew larger and the branches also became shorter and thicker over time. The author also observed the amoeba-like microglia cell, as shown by the yellow arrow, and the microglia with rod-shaped cell body, as shown by the red arrow in Figure 2c. In addition, the qualitative analysis showed that the activation patterns of microglia cells in the injured eye and contralateral eye were different. In the GCL, the branches of microglia in the contralateral eye were more clearly visible than those in the injured eye at day 1 post-ONC (Fig. 2a, c). In the IPL, the branches of microglia cells in the contralateral eye were still slender at day 5 post-ONC, while those in the injured eye were shorter and thicker (Fig. 2a, c).

Secondarily, the quantitative analysis post-ONC was done. In the crushed eye, the total area of microglial cell bodies increased significantly at both day 5 and day 14 post-ONC but not at day 1 post-ONC compared with the normal group (Fig. 2b). In the IPL and OPL, the numbers of microglial cells increased significantly at both day 5 and day 14 post-ONC but not at day 1 post-ONC compared with the normal group (Fig. 2b). In the contralateral eye, the total area of microglial cell bodies increased significantly from day 5 to day 14 post-ONC (Fig. 2d). In the IPL, the numbers of microglial cells had no significant changes during the whole 2-week period post-ONC, while the numbers of microglial cells in the OPL increased significantly at day 5 post-ONC but not at day 1 and day 14 post-ONC (Fig. $2 \mathrm{~d}$ ).

\section{Minocycline treatment delayed the secondary degeneration of RGCs in the contralateral eye}

According to the results of the ONC model, the numbers of RGCs significantly decreased at 5 days post-ONC in both eyes, and the microglial cells were intensively activated at the same time. Therefore, day 5 post-ONC was chosen for the minocycline study in mice. The average densities of RGCs in the left eye of the normal C57 mice, PBS-treated mice (control group) and minocyclinetreated mice were 3,994 $\pm 93 \mathrm{RGCs} / \mathrm{mm}^{2}, 1,681 \pm 115 \mathrm{RGCs} / \mathrm{mm}^{2}$ and $1,808 \pm 72 \mathrm{RGCs} / \mathrm{mm}^{2}$, respectively (Fig. 6a). Compared with the normal group, the densities of RGCs in the PBS group and minocycline group decreased significantly but there was no significant difference between the PBS group and minocycline group (Fig. 6b). In the right contralateral eye, the average densities of RGCs in the normal C57 mice, PBS-treated mice (control group) and minocycline-treated mice were 4,119 $\pm 98 \mathrm{RGCs} / \mathrm{mm}^{2}, 3,075$ $\pm 228 \mathrm{RGCs} / \mathrm{mm}^{2}$ and $3,813 \pm 75 \mathrm{RGCs} / \mathrm{mm}^{2}$, respectively (Fig. $6 a)$. Therefore, the results showed that the densities of RGCs in the minocycline group were significantly higher than those in the PBS group in the contralateral eye (Fig. 6b).

\section{Minocycline treatment reduced the activation of microglia in both eyes}

Microglia cells in the GCL, IPL and OPL of normal mice were characterized by small cell bodies and slender branches, as shown by the white arrow in Figure $3 \mathrm{a}$ and c. Compared with the normal mice, the microglia cells in both eyes of the PBS-treated ONC mice and minocycline-treated ONC mice had larger cell bodies and shorter branches (Fig. 3a, c). This kind of change was similar to that in the retina layers of the mice without PBS or minocycline treatment for day 5 post-ONC (Fig. 2a, c).

The effects of minocycline on microglia activation for 5 days post-ONC in the GCL, IPL and OPL were estimated according to the procedure described in the methods. In the left (crushed) eyes, minocycline treatment inhibited the activation of microglia in the OPL but not in the GCL either IPL compared with the PBS group (Fig. $3 \mathrm{~b}$ ) and the microglia cell density returned to the normal level in the OPL. In the contralateral eye, minocycline treatment also inhibited the activation of microglia in the OPL compared with the PBS group (Fig. 3d) and maintained the microglial cell number in the OPL at the similar level to that in the normal group (Fig. 3d). In the IPL, there was no significant difference in the microglial cell numbers between either two groups in the contralateral eye (Fig. 3d), and this result was similar to that in the IPL of the mice without PBS or minocycline treatment for the 5 days post-ONC (Fig. 2d). These results showed that minocycline could inhibit microglia activation in the OPL in both retinas post-ONC in mice. Although minocycline could not reduce the microglial cell number in the IPL in both eyes, it seemed that the morphology was different between the PBS and minocycline groups for this layer as well as the OPL. Therefore, in order to confirm this issue, detailed quantitative analysis of microglia cell morphology in the IPL and OPL was conducted with the Neurolucida 360 and Neurolucida Explore tools.

The analysis with Neurolucida showed that minocycline significantly reduced the cell body area in the IPL and reduced the microglial cell circumference in the OPL in the left eye (Fig. 4b, d). In the contralateral eye, minocycline decreased the microglial cell circumference and increased the node number, the total number of branches, and the total and the average length of branches of microglia cells in the IPL (Fig. 4f), and reduced microglia cell 
a
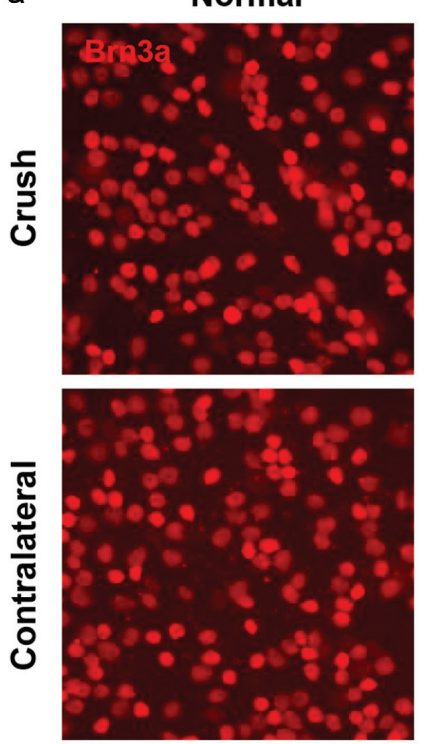

PBS
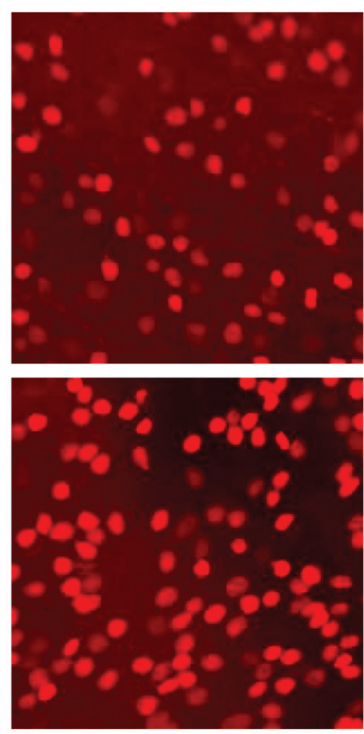

Minocycline
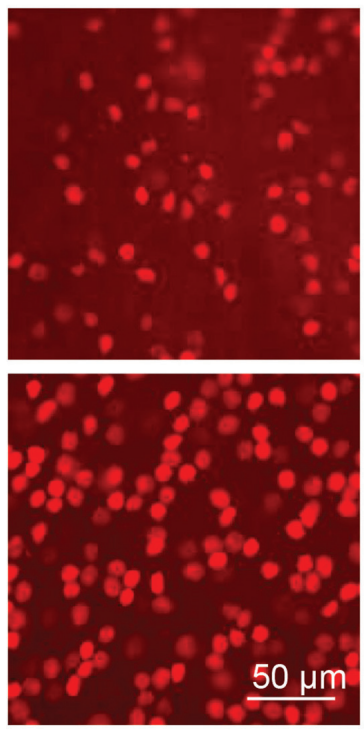

b
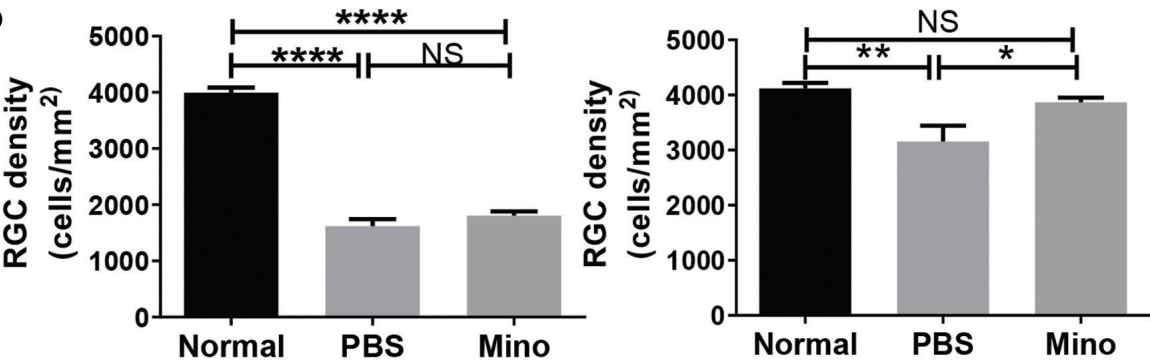

Crush eye

\section{Contralateral eye}

Fig. 6. Changes of the RGCs shown by Brn3a staining in the normal mice, PBS-treated ONC mice and minocycline-treated ONC mice observed under a microscope at a 400x magnification. (a) The typical immunofluorescence staining images of the RGCs in normal mice, PBS-treated ONC mice and PBS-treated ONC mice. The Brn3a-positive cells represent RGCs. (b) The density of RGCs in the crushed eye of mice from the PBS-treated ONC group and the minocyclinetreated ONC group was significantly lower than that in the normal group. There was no significant difference in the density of RGCs between the PBS-treated ONC mice and minocycline-treated ONC mice. The density of RGCs in the contralateral eye of the PBS-treated ONC mice was significantly lower than that in the minocycline-treated ONC mice and the normal mice. There was also no significant difference in the density of RGCs between the minocycline-treated ONC mice and the normal mice. The data were tested by one-way ANOVA followed by Tukey's multiple comparison test. The data are expressed as mean \pm standard error of the mean. NS, no significant difference, ${ }^{*} p<0.05,{ }^{*} p<0.01$, and $* * * * p<0.0001$. The number of animals in the normal group, PBS group and minocycline group were 4, 4 and 7, respectively.

circumference and cell body area in the OPL (Fig. 4h).

\section{Effects of minocycline on the ERG of both eyes}

To examine retinal function in mice, we performed ERG. Retinal neurons in different group of mice responded to light flashes both under dark adapted (scotopic) (Fig. 7a, f) and under light-adapted (photopic) conditions (Fig. 7d, i). In the crushed eye, compared with the normal mice, both a- and b-wave amplitude of scotopic conditions decreased in the PBS-treated mice at 1.0 and $10.0 \mathrm{~cd}$ $\mathrm{s} / \mathrm{m}^{2}$ stimulation. Compared with the normal mice, the a-wave amplitude of scotopic conditions had been decreased in minocycline-treated mice at $1 \mathrm{~cd} \mathrm{~s} / \mathrm{m} 2$ stimulation, the b-wave amplitude of scotopic conditions had been decreased in minocyclinetreated mice at 0.1 and $10.0 \mathrm{~cd} \mathrm{~s} / \mathrm{m}^{2}$ stimulation. There were no significant differences in a-wave and b-wave amplitude between
PBS-treated mice and minocycline-treated mice at any stimulation (Fig. 7b, c, e). These results indicated that the minocycline treatment did not improve the function of photoreceptor cell and bipolar cell in the crushed eye of ONC mice.

In the contralateral eye, compared with the normal mice, the a-wave amplitude of scotopic had been decreased in PBS-treated mice at $1.0 \mathrm{~cd} \mathrm{~s} / \mathrm{m}^{2}$ stimulation, the b-wave amplitude in contralateral eye of scotopic had been decreased in PBS-treated mice at $0.1,1.0$ and $10.0 \mathrm{~cd} \mathrm{~s} / \mathrm{m}^{2}$ stimulation. There were no significant differences in a- and b-wave amplitude between normal mice and minocycline-treated mice at any stimulation. After minocycline treatment, there was a significant difference in a-wave amplitude between minocycline-treated mice and PBS-treated mice at $1.0 \mathrm{~cd}$ $\mathrm{s} / \mathrm{m}^{2}$ stimulation; there was also a significant difference in b-wave amplitude between minocycline-treated mice and PBS-treated mice at $0.1,1.0$ and $10.0 \mathrm{~cd} \mathrm{~s} / \mathrm{m}^{2}$ stimulation (Fig. $7 \mathrm{~g}, \mathrm{~h}, \mathrm{j}$ ). These results indicated that the minocycline-treatment had improved the 
a



Scotopic(cd·s/m²)

PBS

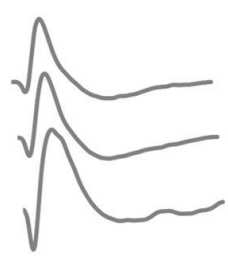

\section{d Photopic (cd $\left.\cdot s / m^{2}\right)$}

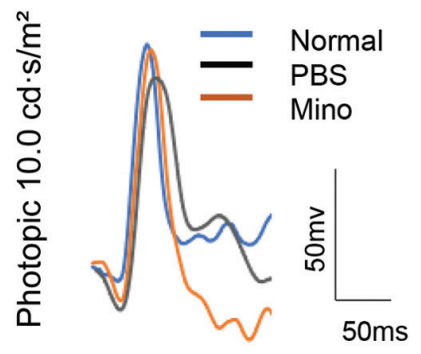


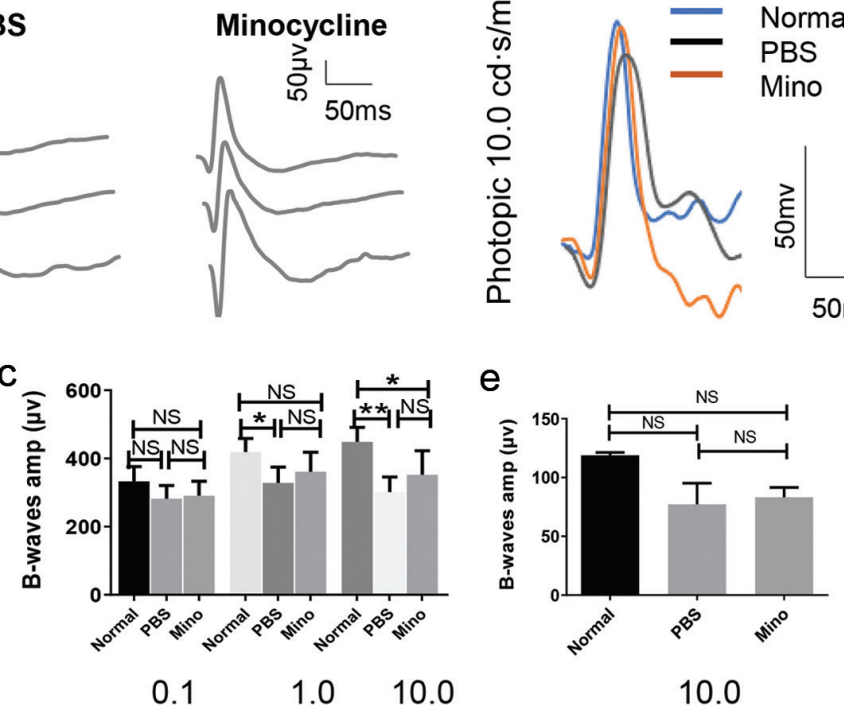

10.0

\section{Crush eye}

f

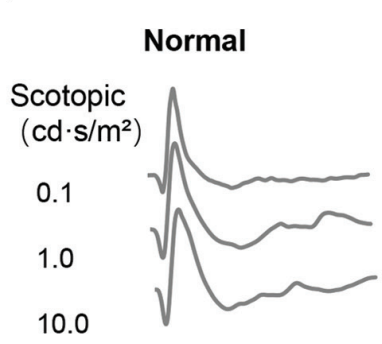

\section{Scotopic $\left(\mathrm{cd} \cdot \mathrm{s} / \mathrm{m}^{2}\right)$ \\ PBS}

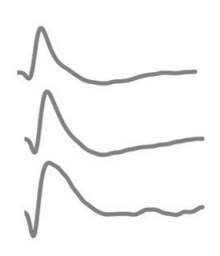

Minocycline

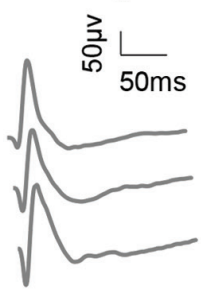

\section{i Photopic (cd.s/m²)}

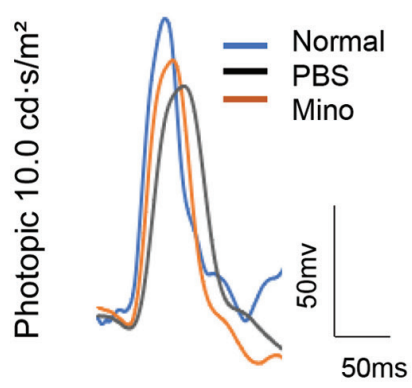

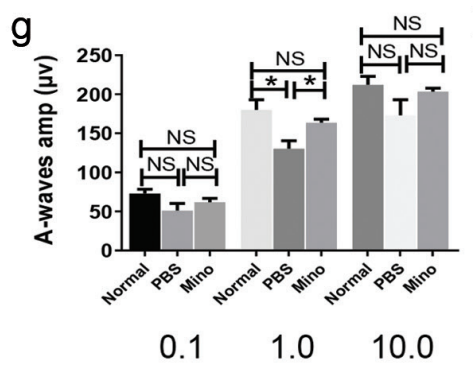
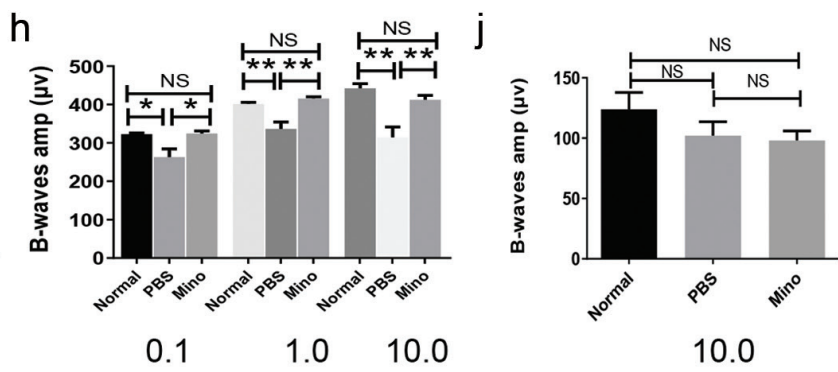

Contralateral eye

Fig. 7. Effects of minocycline on the ERG of both eyes (a) The typical ERG record curve of crushed eye in the normal mice, PBS-treated mice and minocyclinetreated mice under scotopic condition. (b, c) The statistics of a- and b-wave amplitude in the crushed eye of normal mice, PBS-treated mice and minocyclinetreated mice under scotopic condition. (d) The typical ERG record curve of crushed eye in the normal mice, PBS-treated mice and minocycline-treated mice under photopic condition. (e) The statistics of b-wave amplitude in the crushed eye of normal mice, PBS-treated mice and minocycline-treated mice under photopic condition. (f) The typical ERG record curves of contralateral eye in the normal mice, PBS-treated mice and minocycline-treated mice under scotopic condition. ( $\mathrm{g}$ and $\mathrm{h}$ ) The statistics of $\mathrm{a}$ - and b-wave amplitude in the contralateral eye of normal mice, PBS-treated mice and minocycline-treated mice under scotopic condition. (i) The typical ERG record curve of the contralateral eye in the normal mice, PBS-treated mice and minocycline-treated mice under photopic condition. (j) The statistics of b-wave amplitude in the contralateral eye of normal mice, PBS-treated mice and minocycline-treated mice under photopic condition. The data were tested by one-way ANOVA followed by Tukey's multiple comparison test. The data are expressed as mean \pm standard error of the mean. NS, no significant difference. ${ }^{*} p<0.05$ and ${ }^{*} p<0.01$. The number of animals in the normal group, PBS group and minocycline group were 4,4 and 6 , respectively. 
function of photoreceptor cells and bipolar cells in the contralateral eye of ONC mice.

\section{Discussion}

This study confirmed secondary degeneration. First, not only did the number of surviving RGCs significantly decrease in the crushed eye post-ONC but also in the intact contralateral eye. Therefore, the results suggests that secondary degeneration leads to RGC death in the contralateral eye, which is consistent with previous reports of loss of RGCs in the contralateral eye of a glaucoma model. ${ }^{3,27}$ Second, in addition to RGC secondary degeneration, ERG results suggest that photoreceptors and/or bipolar cells also have dysfunction in crushed and contralateral eyes. This is another secondary degeneration confirmation at present study. It is tempting to envision a degenerative cascade in glaucoma, in which a substantial reduction in RGCs leads to final trans-synaptic loss of bipolar cells and finally photoreceptors in retrograde progression. ${ }^{28}$

Activation of microglia may cause secondary degeneration. Indeed, several studies have shown that the contralateral eye also responds to injury by increasing glial cells and altering gene expression. ${ }^{24,29}$

These studies are consistent with the results of immunofluorescence staining observation and manual analysis. After optic nerve compression injury, microglial cells were activated in the operative eye and contralateral eye of mice. This is mainly manifested not only by a significant increase in the number but by morphological changes. The cell body of microglia becomes larger and the branches become shorter and thicker. Some studies have shown that glial responses are transmitted to the contralateral retina through optic crossover in the damaged retina. ${ }^{30}$ In another way, it is suggested that inflammation in one eye can activate RGC fibers and cause inflammation in both superior colliculus, which, in turn, may activate inflammation in the other eye through retrograde transport of RGC fibers. ${ }^{31}$ Due to these controversies, more research and validation are needed to understand how signals from the damaged side are transmitted to the contralateral retina.

As mentioned earlier, minocycline, as a neuroprotective agent, can play a protective role for RGCs in many glaucoma models. In this study, microglial activation of crushed and contralateral eyes was inhibited after minocycline injection, and photoreceptor cells and bipolar cells responded to light strongly. In the contralateral eye, the number of RGCs increased significantly compared with the normal eye, indicating that minocycline protected secondary degeneration of contralateral retinal RGCs after ONC. So, dose this protective effect act directly on the damaged optic nerve by inhibiting microglia or by minocycline? Use of SC560 (a kind of known microglial activation inhibitor) to demonstrate that inhibition of microglial activation protects RGC has suggested that minocycline protects the retina by inhibiting microglial activation and proliferation. ${ }^{25}$

In this study, the author observed the presence of primary and secondary degeneration. Microglia are activated in both the comminuted and contralateral eyes, although the operative eye is more activated than the contralateral one, minocycline has a more significant inhibitory effect on the contralateral eye microglia activation than the surgical eye, the protective effect of secondary degeneration of contralateral eye was more significant. In a rat model with partial transection of the optic nerve, minocycline is ineffective for primary degeneration but effective for secondary degeneration. ${ }^{18}$

Does minocycline protect against secondary degeneration rather than act? This may be related to the dosage used as well as (or) the degree and the type of injury. For example, in an ONC mouse mod$\mathrm{el}$, the role of minocycline in surgical eyes was studied; the results showed that minocycline protected the RGCs of crushed eyes at days 4 and 7 post-ONC, ${ }^{23}$ with $45 \mathrm{mg} / \mathrm{kg}$ intraperitoneal injection given on the first day and $22.5 \mathrm{mg} / \mathrm{kg}$ was subsequently given during the following days. While in my study design, this operation was changed to twice daily with $45 \mathrm{mg} / \mathrm{kg}$ dosage; low doses of minocycline appeared to provide protection in the model of retinal degeneration ischemia-reperfusion injury, and high doses of minocycline showed an injury effect. ${ }^{16}$ In an optic nerve transection model, low and high doses did not play a protective role, ${ }^{32}$ which had used minocycline according to the type and extent of injury and selecting the appropriate dose. The issue should be clarified more clearly when the multiple doses were used in future study.

\section{Future directions}

More insightful experimentation efforts should be exerted to help further explain the potential mechanisms on primary and secondary degeneration. In addition, more precise appropriate dosage of minocycline in neuroprotection should also be discussed, thereby helping the field to achieve a future treatment for RGCs.

\section{Conclusion}

In this study, the author found that minocycline can effectively protect the degeneration of RGCs which died from secondary degeneration in the contralateral eye, although it cannot prevent the primary degeneration of RGCs in the injured eye. This indirectly indicates that the mechanism of secondary degeneration and primary degeneration may be different. It is also possible that the dose used does not prevent more severe primary degeneration.

\section{Acknowledgments}

None.

\section{Data sharing statement}

No additional data are available

\section{Funding}

This study was supported by the National Natural Science Foundation of China (81900264).

\section{Conflict of interest}

The author declares no conflict of interest.

\section{References}

[1] Weinreb RN, Aung T, Medeiros FA. The pathophysiology and treatment of glaucoma: a review. JAMA 2014;311(18):1901-1911. doi:10.1001/jama.2014.3192. 
[2] Li HY, Hong X, Huang M, So KF. Voluntary running delays primary degeneration in rat retinas after partial optic nerve transection. Neural Regen Res 2019;14(4):728-734. doi:10.4103/1673-5374.247481.

[3] Li HY, Ruan YW, Ren CR, Cui Q, So KF. Mechanisms of secondary degeneration after partial optic nerve transection. Neural Regen Res 2014;9(6):565-574. doi:10.4103/1673-5374.130093.

[4] Davis BM, Guo L, Brenton J, Langley L, Normando EM, Cordeiro MF. Automatic quantitative analysis of experimental primary and secondary retinal neurodegeneration: implications for optic neuropathies. Cell Death Discov 2016;2:16031. doi:10.1038/cddiscovery.2016.31.

[5] Liu T, Tatham AJ, Gracitelli CP, Zangwill LM, Weinreb RN, Medeiros FA. Rates of Retinal Nerve Fiber Layer Loss in Contralateral Eyes of Glaucoma Patients with Unilateral Progression by Conventional Methods. Ophthalmology 2015;122(11):2243-2251. doi:10.1016/j. ophtha.2015.07.027.

[6] Sapienza A, Raveu AL, Reboussin E, Roubeix C, Boucher C, Dégardin $\mathrm{J}$, et al. Bilateral neuroinflammatory processes in visual pathways induced by unilateral ocular hypertension in the rat. J Neuroinflammation 2016;13:44. doi:10.1186/s12974-016-0509-7.

[7] Cuenca N, Pinilla I, Fernández-Sánchez L, Salinas-Navarro M, AlarconMartinez L, Aviles-Trigueros $M$, et al. Changes in the inner and outer retinal layers after acute increase of the intraocular pressure in adult albino Swiss mice. Exp Eye Res 2010;91(2):273-285. doi:10.1016/j. exer.2010.05.020.

[8] Jonas M, Cunha BA. Minocycline. Ther Drug Monit 1982;4(2):137145.

[9] Plane JM, Shen Y, Pleasure DE, Deng W. Prospects for minocycline neuroprotection. Arch Neurol 2010;67(12):1442-1448. doi:10.1001/ archneurol.2010.191.

[10] Rosenblat JD, McIntyre RS. Efficacy and tolerability of minocycline for depression: A systematic review and meta-analysis of clinical trials. J Affect Disord 2018;227:219-225. doi:10.1016/j.jad.2017.10.042.

[11] Amani M, Shokouhi G, Salari AA. Minocycline prevents the development of depression-like behavior and hippocampal inflammation in a rat model of Alzheimer's disease. Psychopharmacology (Berl) 2019;236(4):1281-1292. doi:10.1007/s00213-018-5137-8.

[12] Cronin A, Grealy M. Neuroprotective and Neuro-restorative Effects of Minocycline and Rasagiline in a Zebrafish 6-Hydroxydopamine Model of Parkinson's Disease. Neuroscience 2017;367:34-46. doi:10.1016/j.neuroscience.2017.10.018.

[13] Naderi Y, Sabetkasaei M, Parvardeh S, Zanjani TM. Neuroprotective effect of minocycline on cognitive impairments induced by transient cerebral ischemia/reperfusion through its anti-inflammatory and anti-oxidant properties in male rat. Brain Res Bull 2017;131:207-213. doi:10.1016/j.brainresbull.2017.04.010.

[14] Scarabelli TM, Stephanou A, Pasini E, Gitti G, Townsend P, Lawrence $\mathrm{K}$, et al. Minocycline inhibits caspase activation and reactivation, increases the ratio of XIAP to smac/DIABLO, and reduces the mitochondrial leakage of cytochrome $C$ and smac/DIABLO. J Am Coll Cardiol 2004;43(5):865-874. doi:10.1016/j.jacc.2003.09.050.

[15] Hughes EH, Schlichtenbrede FC, Murphy CC, Broderick C, van Rooijen $\mathrm{N}$, Ali RR, et al. Minocycline delays photoreceptor death in the rds mouse through a microglia-independent mechanism. Exp Eye Res 2004;78(6):1077-1084. doi:10.1016/j.exer.2004.02.002.

[16] Huang R, Liang S, Fang L, Wu M, Cheng H, Mi X, et al. Low-dose minocycline mediated neuroprotection on retinal ischemia-reperfusion injury of mice. Mol Vis 2018;24:367-378.

[17] Levkovitch-Verbin H, Kalev-Landoy M, Habot-Wilner Z, Melamed
S. Minocycline delays death of retinal ganglion cells in experimental glaucoma and after optic nerve transection. Arch Ophthalmol 2006;124(4):520-526. doi:10.1001/archopht.124.4.520.

[18] Levkovitch-Verbin H, Spierer O, Vander S, Dardik R. Similarities and differences between primary and secondary degeneration of the optic nerve and the effect of minocycline. Graefes Arch Clin Exp Ophthalmol 2011;249(6):849-857. doi:10.1007/s00417-010-1608-2.

[19] Liu S, Li ZW, Weinreb RN, Xu G, Lindsey JD, Ye C, et al. Tracking retinal microgliosis in models of retinal ganglion cell damage. Invest Ophthalmol Vis Sci 2012;53(10):6254-6262. doi:10.1167/iovs.12-9450.

[20] Nadal-Nicolás FM, Jiménez-López M, Salinas-Navarro M, SobradoCalvo P, Vidal-Sanz M, Agudo-Barriuso M. Microglial dynamics after axotomy-induced retinal ganglion cell death. J Neuroinflammation 2017;14(1):218. doi:10.1186/s12974-017-0982-7.

[21] Levkovitch-Verbin H, Harris-Cerruti C, Groner Y, Wheeler LA, Schwartz M, Yoles E. RGC death in mice after optic nerve crush injury: oxidative stress and neuroprotection. Invest Ophthalmol Vis Sci 2000;41(13):4169-4174.

[22] Heuss ND, Pierson MJ, Roehrich H, McPherson SW, Gram AL, Li L, et al. Optic nerve as a source of activated retinal microglia post-injury. Acta Neuropathol Commun 2018;6(1):66. doi:10.1186/s40478-0180571-8.

[23] Jiao X, Peng Y, Yang L. Minocycline protects retinal ganglion cells after optic nerve crush injury in mice by delaying autophagy and upregulating nuclear factor-KB2. Chin Med J (Engl) 2014;127(9):1749-1754.

[24] Panagis L, Thanos S, Fischer D, Dermon CR. Unilateral optic nerve crush induces bilateral retinal glial cell proliferation. Eur J Neurosci 2005;21(8):2305-2309. doi:10.1111/j.1460-9568.2005.04046.x.

[25] Peng B, Xiao J, Wang K, So KF, Tipoe GL, Lin B. Suppression of microglial activation is neuroprotective in a mouse model of human retinitis pigmentosa. J Neurosci 2014;34(24):8139-8150. doi:10.1523/ JNEUROSCI.5200-13.2014.

[26] Zhang J, Xu D, Ouyang $\mathrm{H}, \mathrm{Hu} \mathrm{S}$, Li A, Luo H, et al. Neuroprotective effects of methyl 3,4 dihydroxybenzoate in a mouse model of retinitis pigmentosa. Exp Eye Res 2017;162:86-96. doi:10.1016/j. exer.2017.07.004.

[27] Kuehn $\mathrm{MH}$. Immune phenomena in glaucoma and conformational disorders: why is the second eye not involved? J Glaucoma 2014;23(8 Suppl 1):S59-S61. doi:10.1097/IJG.0000000000000115.

[28] Calkins DJ. Critical pathogenic events underlying progression of neurodegeneration in glaucoma. Prog Retin Eye Res 2012;31(6):702719. doi:10.1016/j.preteyeres.2012.07.001.

[29] Ananthakrishnan L, Gervasi C, Szaro BG. Dynamic regulation of middle neurofilament RNA pools during optic nerve regeneration. Neuroscience 2008;153(1):144-153. doi:10.1016/j.neuroscience.2008.02.001.

[30] Lucas-Ruiz F, Galindo-Romero C, Rodríguez-Ramírez KT, Vidal-Sanz M, Agudo-Barriuso M. Neuronal Death in the Contralateral Un-Injured Retina after Unilateral Axotomy: Role of Microglial Cells. Int J Mol Sci 2019;20(22):5733. doi:10.3390/ijms20225733.

[31] Macharadze T, Goldschmidt J, Marunde M, Wanger T, Scheich $H_{\text {, }}$ Zuschratter $\mathrm{W}$, et al. Interretinal transduction of injury signals after unilateral optic nerve crush. Neuroreport 2009;20(3):301-305. doi:10.1097/WNR.0b013e32832027e6.

[32] Baptiste DC, Powell KJ, Jollimore CAB, Hamilton C, LeVatte TL, Archibald $\mathrm{ML}$, et al. Effects of minocycline and tetracycline on retinal ganglion cell survival after axotomy. Neuroscience 2005;134(2):575582. doi:10.1016/j.neuroscience.2005.04.011. 